\title{
UNIVERSALLY KURATOWSKI-ULAM SPACES AND OPEN-OPEN GAMES
}

\author{
Piotr Kalemba, Andrzej Kucharski
}

\begin{abstract}
We examine the class of spaces in which the second player has a winning strategy in the open-open game. We show that this spaces are not universally Kuratowski-Ulam. We also show that the games $G$ and $G_{7}$ introduced by P. Daniels, K. Kunen, H. Zhou [Fund. Math. 145 (1994), no. 3, 205-220] are not equivalent.
\end{abstract}

\section{Introduction}

First we shall recall some game introduced in [2] called $G_{2}$. Let $X$ be a topological space equipped with a topology $\mathcal{T}$ and let $\mathcal{B} \subseteq \mathcal{T}$ be its base. The length of the game is $\omega$. Two players I and II take turns playing. At the $n$-th move II chooses a family $\mathcal{P}_{n}$ consisting of open non-empty subset of $X$ such that $\operatorname{cl} \bigcup \mathcal{P}_{n}=X$, then I picks a $V_{n} \in \mathcal{P}_{n}$. I wins iff $\operatorname{cl} \bigcup_{n \in \omega} V_{n}=X$. Otherwise player II wins. Denote by $D_{\text {cov }}$ a collection of families $\mathcal{F}$ consisting of open sets with $\mathrm{cl} \bigcup \mathcal{F}=X$. We say that $\sigma_{\text {cov }}:\left(\bigcup D_{\operatorname{cov}}\right)^{<\omega} \rightarrow D_{\text {cov }}$ is a winning strategy for player $I I$ in the game $G_{2}$ whenever, for any sequence $U_{0}, U_{1}, \ldots$ consisting of non-empty open subsets with $U_{0} \in \sigma_{\text {cov }}(\emptyset)=\mathcal{P}_{0} \in$ $D_{\text {cov }}$ and $U_{n} \in \sigma_{\text {cov }}\left(U_{0}, U_{1}, \ldots, U_{n-1}\right)=\mathcal{P}_{n} \in D_{\text {cov }}$, for all $n \in \omega$, there holds $\operatorname{cl} \bigcup_{n \in \omega} U_{n} \neq X$.

Received: 10.03.2015. Revised: 6.06.2015.

(2010) Mathematics Subject Classification: 54G20, 91A44, 54F99.

Key words and phrases: II-favorable space, uK-U space, tiny sequence.

Andrzej Kucharski thanks European Science Foundation for their support through the grant 3007 within the INFTY program. 
In the paper 22 the authors introduced an open-open game. We say that $G$ is an open-open game of length $\omega$ if two players take turns playing; a round consists of player I choosing a non-empty open set $U \subseteq X$ and player II choosing a non-empty open $V \subseteq U$; I wins if the union of II's open sets is dense in $X$, otherwise II wins. Suppose that there exists a function

$$
s_{o p}: \bigcup\left\{\mathcal{T}^{n}: n \geq 0\right\} \rightarrow \mathcal{T}
$$

such that for each sequence $V_{0}, V_{1}, \ldots$ consisting of non-empty elements of $\mathcal{T}$ with $s_{o p}\left(V_{0}\right) \subseteq V_{0}$ and $s_{o p}\left(V_{0}, V_{1}, \ldots, V_{n}\right) \subseteq V_{n}$, for all $n \in \omega$, there holds $\operatorname{cl} \bigcup_{n \in \omega} V_{n} \neq X$. Then the function $s_{o p}$ is called a winning strategy for II player in the open-open game and we say that the space $X$ is II-favorable.

It is known [2] that the open-open game $G$ is equivalent to $G_{2}$. We consider only games with the length equal to $\omega$. In [2] the authors introduced a game $G_{7}$ which is played as follows: In the $n$-th inning II chooses $\mathcal{O}_{n}$, a family of open sets with $\bigcup \mathcal{O}_{n}$ dense in $X$. I responds with $\mathcal{T}_{n}$, a finite subfamily of $\mathcal{O}_{n}$; I wins if $\bigcup_{n \in \omega} \mathcal{T}_{n}$ is dense subset of $X$; otherwise, II wins.

According to A. Szymański [13] a sequence $\left\{\mathcal{P}_{n}: n \in \omega\right\}$ of open families in $X$ is a tiny sequence if

(1) $\bigcup \mathcal{P}_{n}$ is dense in $X$ for all $n \in \omega$

(2) if $\mathcal{F}_{n}$ is a finite subfamily of $\mathcal{P}_{n}$ for each $n \in \omega$ then $\bigcup\left\{\bigcup \mathcal{F}_{n}: n \in \omega\right\}$ is not dense in $X$.

We call a sequence $\left\{\mathcal{P}_{n}: n \in \omega\right\}$ of open families in $X$ a 1-tiny sequence if (1) $\bigcup \mathcal{P}_{n}$ is dense in $X$ for all $n \in \omega$

(2) if $F_{n}$ is a member of $\mathcal{P}_{n}$ for each $n \in \omega$ then $\bigcup\left\{F_{n}: n \in \omega\right\}$ is not dense in $X$.

M. Scheepers used in the paper [12] negation of the existence of tiny sequence, and 1-tiny sequence - called these properties $S_{\text {fin }}(\mathcal{D}, \mathcal{D})$ and $S_{1}(\mathcal{D}, \mathcal{D})$ respectively. In this paper we refer to notions tiny sequence and 1-tiny sequence, because in some situations (Theorem 1.1 and 1.2 ) we can define them.

Recall another game $G_{4}$ introduced in [2]. In the $n$-th inning player I chooses finite open family $\mathcal{A}_{n}$. Player II responds with a finite, open family $\mathcal{B}_{n}$ with $\left|\mathcal{B}_{n}\right|=\left|\mathcal{A}_{n}\right|$ and for each $V \in \mathcal{A}_{n}$ there exists $W \in \mathcal{B}_{n}$ such that $W \subseteq V$. I wins if $\bigcup_{n \in \omega} \cup \mathcal{B}_{n}$ is dense subset of $X$; otherwise, II wins. One can prove that the game $G_{7}$ is equivalent to the game $G_{4}$ in a way similar to the proof of the equivalence between games $G$ and $G_{2}$.

From now on we consider only c.c.c. spaces.

Theorem 1.1 (M. Scheepers [12, Theorem 2]). II has a winning strategy in the game $G_{7}$ if and only if there exists a tiny sequence. 
Theorem 1.2 (M. Scheepers [12, Theorem 14]). Player II has a winning strategy in the game $G_{2}$ if and only if there exists a 1-tiny sequence.

\section{The main results}

Recall that $X$ is called a II-favorable space if player II a has winning strategy in the game G. If player I has a winning strategy in the game $\mathrm{G}$ then we say that the space is I-favorable.

The following theorem was proven by K. Kuratowski and S. Ulam, see [9]. In order to formulate it, let us recall that: a $\pi$-base is a family of open, nonempty sets such that any open set contains a set from this family, and the $\pi$-weight of a space is the smallest cardinality of a $\pi$-base in this space.

Let $X$ and $Y$ be topological spaces such that $Y$ has countable $\pi$-weight. If $E \subseteq X \times Y$ is a nowhere dense set, then there is a meager set $P \subseteq X$ such that the section $E_{x}=\{y:(x, y) \in E\}$ is nowhere dense in $Y$ for each point $x \in X \backslash P$.

A space $Y$ is universally Kuratowski-Ulam (for short, $u K-U$ space), whenever for a topological space $X$ and a nowhere dense set $E \subseteq X \times Y$ the set

$$
\{x \in X:\{y \in Y:(x, y) \in E\} \text { is not nowhere dense in } Y\}
$$

is meager in $X$, see D. Fremlin [6] (compare [3]). In the paper [7] authors have shown that a compact I-favorable space is universally Kuratowski-Ulam and posed a question: Does there exist a compact universally KuratowskiUlam space which is not I-favorable? We will partially answer to this question, namely we will prove that a II-favorable space is not universally KuratowskiUlam space.

TheOrem 2.1. Let $X$ be a dense in itself space with a $\pi$-base $\mathcal{B}=\bigcup_{n \in \omega} \mathcal{B}_{n}$, where $\mathcal{B}_{n}$ is a maximal family of pairwise disjoint open sets for $n \in \omega$ and let $Y$ be II-favorable c.c.c. space. Then the Kuratowski-Ulam theorem does not hold in $X \times Y$.

Proof. By Theorem 1.2 there is a 1 -tiny sequence $\left\{\mathcal{P}_{n}: n \in \omega\right\}$. Since the space $Y$ satisfies c.c.c., we can assume that each $\mathcal{P}_{n+1}$ is a countable, open, pairwise disjoint family. We can also assume that every $\mathcal{P}_{n+1}$ is a refinement of $\mathcal{P}_{n}$, i.e. each member of $\mathcal{P}_{n+1}$ is a subset of a member of $\mathcal{P}_{n}$. Let $\left\{V_{\sigma}^{n}\right.$ : $\left.\sigma \in{ }^{n} \mathbb{N}\right\}$ be an enumeration of the family $\mathcal{P}_{n}$ such that for each $\tau \in{ }^{n-1} \mathbb{N}$, $\left\{V_{\tau \frown k}^{n}: k \in \mathbb{N}\right\}=\mathcal{P}_{n}$. 
We can assume that $\mathcal{B}_{n+1}$ is a refinement of $\mathcal{B}_{n}$ and $\mid\left\{V \in \mathcal{B}_{n+1}: V \subseteq\right.$ $U\} \mid \geq \omega$ for each $U \in \mathcal{B}_{n}$. For each $n \in \mathbb{N}$ fix a function $f_{n}: \mathcal{B}_{n} \rightarrow{ }^{n} \mathbb{N}$ such that for a fixed $U \in \mathcal{B}_{n}$ we have

$$
\left\{f_{n+1}(V): V \in \mathcal{B}_{n+1} \text { and } V \subseteq U\right\}=f_{n}(U)^{\frown} \mathbb{N} .
$$

Therefore, there holds the condition:

$$
\text { if } V \subset U \text { then } f_{n+1}(V) \supset f_{n}(U) \text { for every } V \in \mathcal{B}_{n+1} \text { and } U \in \mathcal{B}_{n} \text {. }
$$

Consider an open set

$$
F=\bigcup\left\{\bigcup\left\{U \times V_{f_{n}(U)}^{n}: U \in \mathcal{B}_{n}\right\}: n \in \mathbb{N}\right\} .
$$

We shall show that $F$ is dense and $F_{x}=\{y \in Y:(x, y) \in F\}$ is not dense for each $x \in X$. If $x \in X \backslash \bigcap\left\{\bigcup \mathcal{B}_{n}: n \in \mathbb{N}\right\}$ then it is easy to see that $E_{x}$ is not dense. If $x \in \bigcap\left\{\bigcup \mathcal{B}_{n}: n \in \mathbb{N}\right\}$ then by condition (2.2) there is $\sigma \in{ }^{\mathbb{N}} \mathbb{N}$ such that for each $n \in \mathbb{N}$ there exists $U_{n} \in \mathcal{B}_{n}$ with $f_{n}\left(U_{n}\right)=\sigma \mid n$ and $x \in \bigcap\left\{U_{n}: n \in \mathbb{N}\right\}$, hence $F_{x}=\bigcup\left\{V_{\sigma \mid n}^{n}: n \in \mathbb{N}\right\}$. Since $V_{\sigma \mid n}^{n} \in \mathcal{P}_{n}$ for each $n \in \mathbb{N}$ and $\left\{\mathcal{P}_{n}: n \in \omega\right\}$ is a 1-tiny sequence the set $\bigcup\left\{V_{\sigma \mid n}^{n}: n \in \mathbb{N}\right\}$ is not dense.

Now we show that $F$ is a dense set. Let $U \times W$ be any open set. Since $\mathcal{B}$ is a $\pi$-base there are $n \in \mathbb{N}$ and $U_{0} \in \mathcal{B}_{n}$ such that $U_{0} \subseteq U$. Let $\sigma=f_{n}\left(U_{0}\right)$, since $\left\{V_{\sigma \frown k}^{n+1}: k \in \mathbb{N}\right\}$ is a dense family, we get that $W \cap V_{\sigma \frown k}^{n+1} \neq \emptyset$ for some $k \in \mathbb{N}$. By (1), we may take $U_{1} \subseteq U_{0}$ such that $U_{1} \in \mathcal{B}_{n+1}$ and $f_{n+1}\left(U_{1}\right)=\sigma \frown k$. Thus $U_{1} \times V_{f_{n+1}\left(U_{1}\right)}^{n+1} \cap U \times W \neq \emptyset$.

Since $\mathbb{R}$ with natural topology satisfies assumption of the above theorem and every universally Kuratowski-Ulam space is c.c.c. space, we get the following theorem.

TheOREm 2.2. A II-favorable space is not universally Kuratowski-Ulam space.

Following [10, pp. 86-91] recall category measure space. If $X$ is a topological space with finite measure $\mu$ defined on the $\sigma$-algebra $S$ of sets having the Baire property, and if $\mu(E)=0$ if and only if $E$ is of a meager set, then $(X, S, \mu)$ is called a category measure space. An example of a regular Baire space which is a category measure space, is an open interval $(0,1)$ with Lebesgue measure $\mu_{l}$ and density topology $\mathcal{T}_{d}$, see [10. For density topology and measurable set $A \subseteq(0,1)$ the following conditions are equivalent: 
(1) $\mu_{l}(A)=0$,

(2) $A$ is closed and nowhere dense.

In the space $\left((0,1), \mathcal{T}_{d}\right)$ there is a 1-tiny sequence but there is no tiny sequence. Indeed, define a 1-tiny sequence in the following way: let $\mathcal{P}_{n}=\{U: U \in$ $\mathcal{T}_{d}$ and $\left.\mu_{l}(U) \leq \frac{1}{3^{n}}\right\}$. If $\left\{U_{n}: n \in \mathbb{N}\right\}$ is a family chosen by player I then $\mu_{l}\left(\bigcup\left\{U_{n}: n \in \mathbb{N}\right\}\right) \leq \frac{1}{2}$. Therefore $\left\{U_{n}: n \in \mathbb{N}\right\}$ is not a dense family. Now assume that there exists a tiny sequence $\left\{\mathcal{P}_{n}: n \in \mathbb{N}\right\}$. In each stage we choose a finite subfamily $\mathcal{R}_{n} \subset \mathcal{P}_{n}$ such that $\mu_{l}\left(\bigcup\left\{\bigcup \mathcal{R}_{i}: i \leq n\right\}\right) \geq 1-\frac{1}{n}$, hence we get a dense family $\bigcup\left\{\mathcal{R}_{n}: n \in \mathbb{N}\right\}$.

The authors of the paper [2] posed a question (Question 4.3): Does a player have a winning strategy in the game $G$ if and only if the same player has a winning strategy in the game $G_{7}$. The author of paper [12] showed that if $\operatorname{cov}(\mathcal{M})<\mathfrak{d}$ the answer is NO. We show that games $G$ and $G_{7}$ are not equivalent.

Corollary 2.3. The game $G$ is not equivalent to the game $G_{7}$.

Proof. By Theorem 1.2 a winning strategy of II player in the game $G$ is equivalent to the existence of a 1-tiny sequence and by Theorem 1.1 the existence of a winning strategy of player II in the game $G_{7}$ is equivalent to the existence of a tiny sequence. Since in the space $\left((0,1), \mathcal{T}_{d}\right)$ there is a 1-tiny sequence but there is no tiny sequences we get that games $G$ and $G_{7}$ are not equivalent.

Since the game $G_{7}$ is equivalent to the game $G_{4}$, we get the following:

Corollary 2.4. The game $G$ is not equivalent to the game $G_{4}$.

\section{Some remarks}

It is known that on the $\omega_{1}$ with discrete topology II player has a winning strategy in the game $G_{7}$, but one can pose a question:

Is it possible to construct a tiny sequence $\left\{\mathcal{P}_{n}: n \in \omega\right\}$ on a discrete space of the size $\omega_{1}$ with $\left|\mathcal{P}_{n}\right|=\omega$ for all $n \in \omega$ ?

The following Remark 3.1 gives us the answer - it is possible if and only if the dominating number is equal $\omega_{1}$. This is reformulation of well know results about critical cardinal number, see W. Just, A.W. Miller, M. Scheepers and P.J. Szeptycki [5]; D. Fremlin, A.W. Miller [4] and B. Tsaban [14].

Recall that $f \leq^{*} g$ denotes that for almost all $n \in \omega$ holds $f(n) \leq g(n)$, where $f, g$ are functions defined on natural numbers. A family $\mathcal{R} \subseteq{ }^{\omega} \omega$ is 
a dominating family if for each $f \in{ }^{\omega} \omega$ there is $g \in \mathcal{R}$ such that $f \leq^{*} g$. The dominating number $\mathfrak{d}$ is the smallest cardinality of a dominating family:

$$
\mathfrak{d}=\min \{|\mathcal{R}|: \mathcal{R} \text { is dominating }\} .
$$

REMARK 3.1. The smallest cardinality $\kappa$ such that there exists a tiny sequence $\left\{\mathcal{P}_{n}: n \in \omega\right\}$ on the discrete space of the size $\kappa$ with $\left|\mathcal{P}_{n}\right|=\omega$ for all $n \in \omega$ is equal to $\mathfrak{d}$.

Proof. Let $X$ be any discrete space for which there exists a tiny sequence $\left\{\mathcal{P}_{n}: n \in \omega\right\}$. We can assume that every $\mathcal{P}_{n}$ is a partition of $X$ into countably many blocks $\left\{X_{0}^{n}, X_{1}^{n}, \ldots\right\}$, so we may define for each $x \in X$ a function $f_{x}: \omega \rightarrow \omega$ in the following way: $f_{x}(n)=k$ whenever $x \in X_{k}^{n}$. Take an arbitrary function $f: \omega \rightarrow \omega$, and any $x \in X \backslash \bigcup\left\{\bigcup\left\{X_{k}^{n}: k \leq f(n)\right\}: n<\omega\right\}$, then $f$ is dominated by the function $f_{x}$. It shows that $\left\{f_{x}: x \in X\right\}$ is a dominating family, hence $|X| \geq \mathfrak{d}$.

Now, let $\mathcal{F} \subset \omega^{\omega}$ be a dominating family of the cardinality $\mathfrak{d}$. Without loss of generality assume that for each function $f: \omega \rightarrow \omega$ there is $g \in \mathcal{F}$ such that $f(n)<g(n)$ for all $n<\omega$. We treat $\mathcal{F}$ as a discrete topological space. For $n, k \in \omega$ put $A_{k}^{n}=\{f \in \mathcal{F}: f(n) \leq k\}$ and set $\mathcal{P}_{n}=\left\{A_{k}^{n}: k<\omega\right\}$. Of course, each family $\mathcal{P}_{n}$ is increasing and has the union equal to $\mathcal{F}$. From each $\mathcal{P}_{n}$ take some single $A_{f(n)}^{n}$ where $f: \omega \rightarrow \omega$. If $\bigcup\left\{A_{f(n)}^{n}: n<\omega\right\}$ was equal to $\mathcal{F}$, then it would contain such a function $g$ that $g(n)>f(n)$ for all $n \in \omega$, but it is not the case. Therefore $\left\{\mathcal{P}_{n}: n \in \omega\right\}$ is a tiny sequence.

Recall a definition of a Baire number $\operatorname{cov}(\mathcal{M})$ for the ideal $\mathcal{M}$ of meager subsets of real line $\mathbb{R}$ :

$$
\operatorname{cov}(\mathcal{M})=\min \{|\mathcal{A}|: \mathcal{A} \subseteq \mathcal{M} \text { and } \bigcup \mathcal{A}=\mathbb{R}\} .
$$

T. Bartoszyński [1] proved that $\operatorname{cov}(\mathcal{M})$ is the cardinality of the smallest family $\mathcal{F} \subseteq{ }^{\omega} \omega$ such that

$$
\forall\left(g \in{ }^{\omega} \omega\right) \exists(f \in \mathcal{F}) \forall(n \in \omega) f(n) \neq g(n) .
$$

We get another well known characterization of such families by a 1-tiny sequence.

REMARK 3.2. The smallest cardinality $\kappa$ such that there exists a 1-tiny sequence $\left\{\mathcal{P}_{n}: n \in \omega\right\}$ on the discrete space of the size $\kappa$ with $\left|\mathcal{P}_{n}\right|=\omega$ for all $n \in \omega$ is equal to $\operatorname{cov}(\mathcal{M})$. 
We give the proof for the sake of completeness. We shall prove that the smallest cardinality of a family $\mathcal{F} \subseteq{ }^{\omega} \omega$ such that

$$
\forall\left(g \in{ }^{\omega} \omega\right) \exists(f \in \mathcal{F}) \forall(n \in \omega) f(n) \neq g(n)
$$

is equal to the smallest cardinality $\kappa$ such that there exists a 1-tiny sequence $\left\{\mathcal{P}_{n}: n \in \omega\right\}$ on the discrete space $\kappa$ with $\left|\mathcal{P}_{n}\right|=\omega$ for all $n \in \omega$.

Proof. Let $\mathcal{F}=\left\{f_{\alpha}: \alpha<\kappa\right\} \subseteq{ }^{\omega} \omega$ be a family with the property $(*)$. Define $A_{n}^{i}=\{f \in \mathcal{F}: f(i)=n\}$ for every $i, n \in \omega$. Let $\mathcal{P}_{i}=\left\{A_{n}^{i}: n \in \omega\right\}$ for $i \in \omega$. We will show that $\left\{\mathcal{P}_{i}: i \in \omega\right\}$ is a 1-tiny sequence. Assume that we have chosen $A_{n_{i}}^{i} \in \mathcal{P}_{i}$ for each $i \in \omega$. Define a function $g(i)=n_{i}$ for $i \in \omega$. Since $\mathcal{F}$ satisfies $(*)$ there is $f \in \mathcal{F}$ such that $f(i) \neq g(i)$ for each $i \in \omega$. Therefore we get $f \in \mathcal{F} \backslash \bigcup\left\{A_{n_{i}}^{i}: i \in \omega\right\}$.

Let $\left\{\mathcal{P}_{n}: n \in \omega\right\}$ be a 1-tiny sequence with $\left|\mathcal{P}_{n}\right|=\omega$ and $\bigcup \mathcal{P}_{n}=\kappa$ for each $n \in \omega$. We can assume that each $\mathcal{P}_{n}$ consists of pairwise disjoint subsets of $\kappa$. Let $\left\{A_{k}^{n}: k \in \omega\right\}$ be a enumeration of $\mathcal{P}_{n}$. We define a function $f_{x} \in{ }^{\omega} \omega$ for each $x \in \kappa$ in the following way: $f_{x}(i)=n$, where $x \in A_{n}^{i}$ for each $i \in \omega$. The family $\left\{f_{x}: x \in \kappa\right\}$ satisfies $(*)$. Indeed, let $g \in{ }^{\omega} \omega$ be any function. Since $\left\{\mathcal{P}_{n}: n \in \omega\right\}$ is a 1-tiny sequence, choose $x \in \kappa \backslash \bigcup\left\{A_{g(i)}^{i}: i \in \omega\right\}$. Finally, observe that $f_{x}(i) \neq g(i)$ for every $i \in \omega$.

We shall recall definition of the bounding number

$$
\left.\mathfrak{b}=\min \left\{|\mathcal{F}|: \mathcal{F} \subseteq{ }^{\omega} \omega \text { and } \forall\left(g \in{ }^{\omega} \omega\right) \exists(f \in \mathcal{F}) \neg\left(f \leq^{*} g\right)\right)\right\} .
$$

We say that a sequence $\left\{\mathcal{P}_{n}: n \in \omega\right\}$ of open families in $X$ is a $\mathfrak{b}$-tiny sequence if

(1) $\bigcup \mathcal{P}_{n}$ is dense in $X$ for all $n \in \omega$;

(2) if $\mathcal{F}_{n}$ is a finite subfamily of $\mathcal{P}_{n}$ for each $n \in \omega$, then there exists strictly increasing sequence $\left\{n_{i}: i \in \omega\right\}$ such that

$$
\bigcup\left\{\bigcup \mathcal{F}_{n_{i}}: i \in \omega\right\}
$$

is not dense in $X$.

We get the next reformulation of the bounding number.

REMARK 3.3. The smallest cardinality $\kappa$ such that there exists a $\mathfrak{b}$-tiny sequence $\left\{\mathcal{P}_{n}: n \in \omega\right\}$ on the discrete space of the size $\kappa$ with $\left|\mathcal{P}_{n}\right|=\omega$ for all $n \in \omega$ is equal to $\mathfrak{b}$. 
Acknowledgement. The authors are indebted to the Referee for very careful reading of the paper and valuable comments.

\section{References}

[1] Bartoszyński T., Combinatorial aspects of measure and category, Fund. Math. 127 (1987), no. 3, 225-239.

[2] Daniels P., Kunen K., Zhou H., On the open-open game, Fund. Math. 145 (1994), no. 3, 205-220.

[3] Fremlin D., Natkaniec T., Recław I., Universally Kuratowski-Ulam spaces, Fund. Math. 165 (2000), no. 3, 239-247.

[4] Fremlin D., Miller A.W., On some properties of Hurewicz, Menger and Rothberger, Fund. Math. 129 (1988), 17-33.

[5] Just W., Miller A.W., Scheepers M., Szeptycki P.J., The combinatorics of open covers (II), Topology Appl. 73 (1996), 241-266.

[6] Fremlin D., Universally Kuratowski-Ulam spaces, a note from: http://www.essex.ac. uk/maths/staff/fremlin/preprints.htm

[7] Kucharski A., Plewik S., Game approach to universally Kuratowski-Ulam spaces, Topology Appl. 154 (2007), no. 2, 421-427.

[8] Kucharski A., Plewik S., Inverse systems and I-favorable spaces, Topology Appl. 156 (2008), no. 1, 110-116.

[9] Kuratowski K., Ulam S., Quelques propriétés topologiques du produit combinatoire, Fund. Math. 19 (1932), 247-251.

[10] Oxtoby J., Measure and category, Springer-Verlag, New York, 1971.

[11] Pawlikowski J., Undetermined sets of point-open games, Fund. Math. 144 (1994), 279285.

[12] Scheepers M., Combinatorics of open covers (V): Pixley-Roy spaces of sets of reals, and $\omega$-covers., Topology Appl. 102 (2000), no. 1, 13-31.

[13] Szymański A., Some applications of tiny sequences, in: Proceedings of the 11th winter school on abstract analysis (Železna Rudá, 1983), Rend. Circ. Mat. Palermo (2) 1984, Suppl. no. 3, pp. 321-328.

[14] Tsaban B., Strong $\gamma$-sets and other singular spases, Topology Appl. 153 (2005), 620639.

Institute of Mathematics

University of Silesia

BANKOWA 14

40-007 Katowice

POLAND

e-mail: piotr.kalemba@us.edu.pl

e-mail: akuchar@math.us.edu.pl 